\title{
The Coronal Emission-Line Region in AGNs: A Hubble Space Telescope View
}

\author{
X. Mazzalay ${ }^{1,2}$, A. Rodríguez-Ardila ${ }^{3}$, and S. Komossa ${ }^{1}$ \\ ${ }^{1}$ Max-Planck-Institut für extraterrestrische Physik, \\ Postfach 1312, 85741 Garching, Germany \\ Email: ximena, skomossa@mpe.mpg.de \\ ${ }^{2}$ Instituto de Astronomía Teórica y Experimental, CONICET-UNC, \\ Laprida 854, X5000BGR, Córdoba, Argentina \\ ${ }^{3}$ Laboratório Nacional de Astrofísica, \\ Rua dos Estados Unidos 154, Bairro das Nacöes, CEP 37500-000, Itajubá, MG, Brazil \\ Email: aardila@lna.br
}

\begin{abstract}
We present a study of HST/STIS optical spectra of a sample of ten Seyfert galaxies aimed at analyzing the structure and physical properties of the coronal line region. The high spatial resolution provided by STIS (about $7 \mathrm{pc}$ for the closest objects) allowed us to resolve the coronal line region and obtain key information about the kinematics of the coronal-line gas, measure directly its spatial scale, and study the mechanisms that drive the high-ionization lines.
\end{abstract}

Keywords. galaxies: active, galaxies: nuclei, line: formation, galaxies: kinematics and dynamics

Coronal lines (CLs) are collisionally excited forbidden transitions within low-lying levels of highly ionized species (ionization potential IP $\geqslant 100 \mathrm{eV}$ ) and their presence indicates the existence of very energetic processes associated with the nuclear activity. Although they have been long known to be a common feature in the spectra of AGN, a clear picture that explains the formation of the CLs is far from complete. In order to have a better understanding of the so-called coronal line region (CLR) we analyzed HST/STIS optical spectra of ten nearby Seyfert galaxies. The lines chosen for this analysis range from $[\mathrm{Ne} \mathrm{V}]$ and [Fe VII], with ionization potential of about $100 \mathrm{eV}$, to very high ionization lines like [Fe XIV] $(\mathrm{IP}=361 \mathrm{eV})$ and [S XII] $(\mathrm{IP}=504 \mathrm{eV})$. We found that CLs can be emitted from very compact regions $(<10 \mathrm{pc})$ up to scales of hundreds of parsecs. There is a trend for CLs to follow the same spatial distribution and kinematics as the lower ionization lines. A particularly interesting case is the [Fex] line displayed by Mrk 573 . This high-ionization line extends up to $120 \mathrm{pc}$ from the nucleus. This extent is remarkable because it has been rarely observed before. This line also displays a double-peak structure, with the peak separation increasing when going from one side of the nucleus to the other. ¿From a direct comparison between the radio and the CL emission we found that neither the strength nor the kinematics of the CLs scale in any obvious and strong way with the radio jets. Moreover, the similarity of the flux distributions and kinematics of the CLs and low-ionization lines, the low temperatures derived for the gas, and the success of photoionization models to reproduce, within a factor of few, the observed line ratios, point towards photoionization as the main driving mechanism of CLs. The complete results from our analysis are presented by Mazzalay et al. (2009).

\section{Reference}

Mazzalay, X., Rodríguez-Ardila, A., \& Komossa, S. 2009, submitted to MNRAS 